IOS Press

\title{
Thesis
}

\section{Behaviour recognition in smart homes}

\author{
Sook-Ling Chua \\ School of Engineering and Advanced Technology, Massey University, New Zealand \\ E-mail: linda.slchua@gmail.com
}

Keywords: Behaviour recognition, activity segmentation, sensor selection

\section{Problem addressed}

One application of smart homes is to take sensor activations from a variety of sensors around the house and use them to recognise the particular behaviours of the inhabitants. There are many reasons to recognise human behaviours; one of the reasons being to monitor the elderly or cognitively impaired who are living alone.

The thesis views the behaviour recognition problem as a task of mapping the sensory outputs to a sequence of activities performed by the inhabitant. The main focus is the development of machine learning methods to find an approximation to the mapping between sensor outputs and behaviours that is therefore suitable to be used in a smart home to monitor the inhabitant's behaviours. However, data from a sensor stream consists of an unending sequence of sensor readings. This means that the start and end of an activity are unknown. It is important that the recognition system has the ability to perform segmentation and behaviour recognition simultaneously on the sensory stream. The thesis investigates two approaches to address the behaviour recognition problem: one using a supervised learning on a labelled sensor streams and one using an unsupervised learning on unlabelled sensor streams. This includes both the segmentation of the sensor stream into suitable patterns and the identification of patterns that correspond to human behaviours.

The thesis also addresses the sensor selection problem through an information-theoretic approach, which is based on information gain, modelled in the form of a decision tree. The main idea is to identify the set of informative sensors that could provide the most information about the inhabitant's behaviours.

The efficacy of the methods was demonstrated with experiments on a real smart home dataset obtained from the MIT PlaceLab and other benchmark datasets. The methods were also compared with other learning algorithms. The results obtained from her work showed that we can now recognise normal behaviours of somebody living in a smart home.

The author successfully defended her thesis entitled Behaviour Recognition in Smart Homes at Massey University, New Zealand on August 21st, 2012. Her supervisors were Prof. Hans W. Guesgen and Assoc. Prof. Dr. Stephen Marsland. The examiner committee was composed of Dr. Patricia Riddle (University of Auckland), Mr. Paul Lyons (Massey University) and Prof. Diane Cook (Washington State University) as the external examiner. Prof. Margie Comrie acted as the convenor.

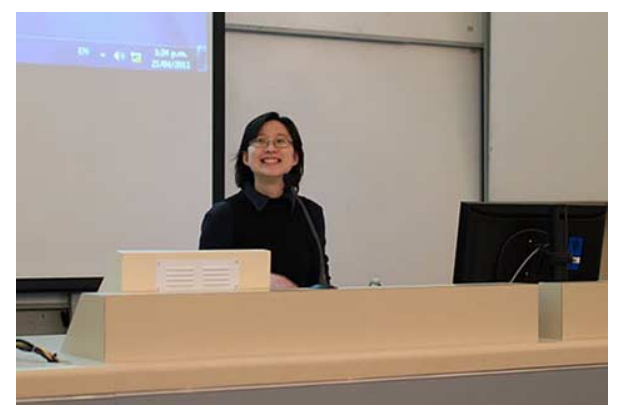

Fig. 1. The author during the New Zealand Computer Science Research Student Conference (NZCSRSC), where she was awarded the best student presentation. 